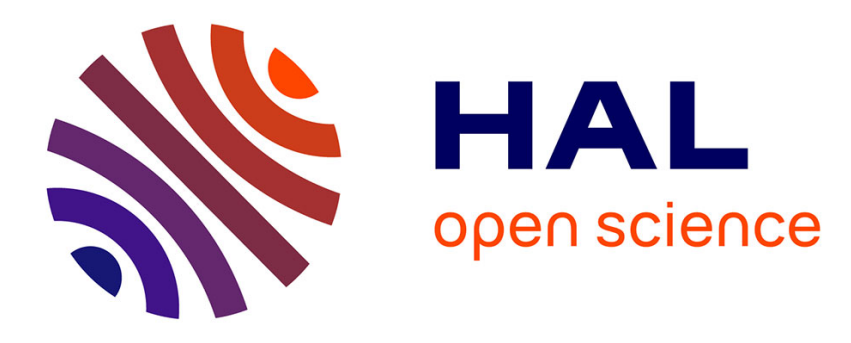

\title{
Une tentative de transformation des études sur les légendes contemporaines en propagande négationniste
}

\author{
Véronique Campion-Vincent
}

\section{To cite this version:}

Véronique Campion-Vincent. Une tentative de transformation des études sur les légendes contemporaines en propagande négationniste. Le monde alpin et rhodanien, 2002. halshs-01698201

\section{HAL Id: halshs-01698201 https://shs.hal.science/halshs-01698201}

Submitted on 5 Feb 2018

HAL is a multi-disciplinary open access archive for the deposit and dissemination of scientific research documents, whether they are published or not. The documents may come from teaching and research institutions in France or abroad, or from public or private research centers.
L'archive ouverte pluridisciplinaire HAL, est destinée au dépôt et à la diffusion de documents scientifiques de niveau recherche, publiés ou non, émanant des établissements d'enseignement et de recherche français ou étrangers, des laboratoires publics ou privés. 


\section{Une tentative de transformation des études sur les légendes contemporaines en propagande négationniste*}

\section{L'OUVRAGE}

Arthur Ponsonby (1871-1946) était membre élu du parlement britannique, annobli après 1930 (Lord Ponsonby). Il fut l'auteur d'un livre demeuré disponible depuis sa première publication en 1928. Intitulé Falsehood in War-Time: containing an assortment of lies circulated throughout the nations during the Great War cet ouvrage est un assortiment décousu d'exemples de fausses informations et de leur promotion par les services de propagande des alliés. Issu d'un milieu aristocratique, Arthur Ponsonby passa du libéralisme au socialisme et au pacifisme. Ensemble indigné d'études de cas, son ouvrage doit être lu comme une œuvre de propagande pacifiste. D'autres travaux de niveau populaire (Graux 1918-1920) ou érudit (Bloch 1921; Dauzat 1918, 1919; Van Langenhove 1917) publiés en français s'étaient fixé un programme analogue. L'étude de Marc Bloch, qui rend compte de façon détaillée de ces trois travaux et y ajoute des commentaires originaux et pénétrants tirés de son expérience au front, est une lecture recommandée ${ }^{(1)}$.

L’ouvrage de Ponsonby a été republié par les négationnistes ${ }^{(2)}$ : en 1980 et 1991 aux États-Unis, en 1996 en France ${ }^{(3)}$, avec une préface, une traduction et des notes de Jean Plantin ${ }^{(4)}$ qui ajoute au titre original le terme de «rumeurs" Mensonges et rumeurs en temps de guerre. Deux points sont à considérer dans cette publication (non disponible dans le commerce puisque la diffusion de publications négationnistes est illégale en France depuis l'adoption de la loi Gayssot ${ }^{(5)}$ le 13 juillet 1990): sa préface et sa bibliographie.

* Ce texte est la traduction de la note publiée dans Letters to Ambrose Merton, A Quarterly Folklotre Miscellany
9, mars 1997.

(1) Les travaux menés depuis 1992 par les historiens regroupés autour de l'Historial de la Grande Guerre à Péronne ont renouvelé la problématique de ces questions, qui tournent autour de la nature des atrocités attribuées aux Allemands en 1914: réalité, propagande, rumeurs? Je n'ai pu encore consulter l'ouvrage récent d'Horne et Kramer (2001) qui se situe dans cette mouvance, et dont l'enquête historique conclut à
la réalité de bien des faits atroces.

(2) Ceux-ci se désignent, on le sait comme révisionnistes, toutefois on emploiera le terme négationniste qui les décrit avec plus de précision et d'exactitude. En effet ils nient la réalité des chambre à gaz et, au delà, de
la volonté de génocide des juifs chez les nazis.

(3) La Bibliothèque nationale de France catalogue une publication en langue française de cet ouvrage, édité à Bruxelles (sans date ni mention d'un traducteur) sous le titre Faussaires en temps de guerre.

(4) Auquel on se réferera par la suite comme J.-P.

(5) La loi précise que la négation des crimes contre l'humanité (tels qu'ils ont été définis dans les Statuts du Tribunal de guerre de Nuremberg publiés en annexe à l'accord de Londres du 8 août 1945) est punissable. 


\section{LA PRÉFACE DE JEAN PLANTIN}

Pages XIII-XIV, J.-P. fait l'éloge de l'ouvrage de Ponsonby pour son étude approfondie de "l'histoire de l'usine de transformation en graisse de cadavres humains" [ce chapitre occupe les pages 117-130]. Il se demande si la propagande a créé ce pseudo-fait ou si la propagande s'est appuyée sur des rumeurs et légendes déjà existantes. Affirmant: "On connaît la présence dans le folklore européen et latino-américain, par exemple, de récits légendaires qui évoquent l'extraction de graisse à partir de cadavres humains", il signale alors deux articles, l'un dont je suis l'auteur (Campion-Vincent $\left.1994^{(6)}\right)$, l'autre écrit par Joisten \& Chanaud en $1992^{(7)}$.

Cette référence ignore le propos de nos articles: nous présentions des accusations contre des sorciers qui, affirmait-on, extrayaient la graisse de leurs victimes afin de créer du verre de qualité (Joisten \& Chanaud) ou contre le pishtaco, un personnage vampiresque fantasmatique, présent dans le folklore andin depuis le XVTe siècle ${ }^{(8)}$. Si ces accusations peuvent sembler plausibles à ceux qui croient à l'existence des sorciers ou du pishtaco, elles renvoient aux qualités surnaturelles prêtées à ces deux groupes et ne sont donc nullement comparables à des allégations d'actions conduites par des ennemis durant une guerre.

Pages XIV-XV, bien que Ponsonby n'en fasse pas mention, J.-P. discute et dénonce la rumeur concernant « les gazages massifs d'êtres humaine qui auraient eu lieu en Serbie aux alentours de 1915-1917 ». Pages XV-XVII, J.-P. embraye sur son sujet central "cette histoire de gazage a resurgi pendant la Seconde Guerre mondiale» et dénonce l'histoire « un ensemble complexe d'interaction entre rumeurs et propagande" comme ayant été totalement discrédité, naturellement, par les négationnistes.

\section{LA BIBLIOGRAPHIE DE JEAN PLANTIN}

Environ $80 \%$ de la bibliographie (pages 249-256) viennent de sources négationnistes, identifiables par leurs revues (Annales d'histoire révisionniste, Journal of Historical Review, Revue d'histoire révisionniste) ou maisons d'édition (Toronto: Samizdat publishers; Paris: La Vieille Taupe). Quelques références, généralement utilisées par J.-P. pour ses annotations, concernent des études historiques et des livres, de niveau populaire ou érudit, sur la propagande.

La bibliographie contient plusieurs références sur les légendes contemporaines. Non utilisées dans les commentaires et notes de J.-P., ces références remplissent à l'évidence une fonction de légitimation. On y trouve les cinq ouvrages écrits par Jan Harold Brunvand sur les légendes urbaines (1981, 1984, 1986, 1989, 1993), deux publications de Campion-Vincent (1992b, 1994) deux publications de Campion-Vincent \& Renard (1990,1992) deux études américaines sur la définition des légendes contemporaines de Linda Degh (1991) et Jeffrey Victor (1993). J.-P. cite également des auteurs français sur les rumeurs: Marie Bonaparte (1946), Jean-Noël Kapferer (1987), Jean Loïc Le Quellec

(6) Cette communication à un colloque reprenait Campion-Vincent 1992a, plus accessible.

(7) Le sujet traité par Joisten \& Chanaud (1992) a été ultérieurement repris et développé dans †C. Joisten, R. Chanaud \& A. Joisten (1992), cf. 36-43 et 126-141. Voir dans le présent numéro une actualisation de ces recherches (articles de Raymond Moyroud et de C. Abry et A. Joisten).

(8) Le pishtaco est un étranger, hier moine mendiant, doué de pouvoirs surnaturels qu'il utilise pour extraire des Indiens qu'il rencontre cheminant sur les routes à la nuit tombée leur graisse qui servait autrefois à fondre les cloches, et aujourd'hui à lubrifier les ordinateurs. 
(1991), Edgar Morin (1969), Françoise Reumaux (1994, 1996), Michel-Louis Rouquette (1975). Cette stratégie d'appropriation est particulièrement nette dans la section "adresses utiles", section où l'ISCLR [International Society for Contemporary Legend Research, fondée en 1988 et publiant la revue Contemporary Legend] et le bulletin Tutte Storie figurent à côté de publications négationnistes aux États-Unis, en Allemagne et en France.

256

BIBLIOGRAPHIE

\section{AdRESSES UTILES}

* Journal of Historical Rewiev, Institute for Historical Review, P.O. Box 2739, Newport Beach, CA 92659, Étas-Unis.

* Historische Tatsachen, Verlag für Volkstum und Zeitgeschichtsforschung, Postfach 1643, 32590 Vlotho/Weser, Allemagne.

* Annales d'histoire révisionniste, BP 9805, 75224 Paris CEDEX 05 (8 numéros parus, soit environ 1500 pages de texte; revue interdite de vente aux mineurs en France).

* Revue d'histoire révisionniste, BP 122, 92704 Colombes CEDEX (6 numéros parus, soit environ 1200 pages de texte; revue interdite de vente aux mineurs en France).

* International Society for Contemporary Legend Research (I.S.C.I.R.); publie la lettre d'information Foaftale News (environ 3 numéros par an) et Contemporary Legend (revue annuelle). On peut s'abonner (18 dollars américains pour 1996) auprès de Paul SMITH, Department of Folklore, Memorial University of Newfoundland, St John's, Newfoundland, A1B 3X8, Canada.

* Tutte storie, bulletin du Centro per la Raccolta delle Voci e Leggende Contemporanee, Casella Postale 53, 15000 Alessandria, Italie.

\section{De l'édition de Plantin}

J'avoue que cela a été une surprise désagréable de découvrir ces citations et, au delà de mon cas personnel, cette utilisation de notre travail comme légitimation par un mouvement avec lequel aucun d'entre nous n'a, me semble-t-il, d'affinité. Mais ceci n'est qu'un exemple d'un malentendu profond qui mène souvent à des difficultés, par exemple dans les relations avec les médias. Nombreux sont ceux qui voient les spécialistes des légendes comme dotés de l'unique, mais utile, fonction de dénoncer les légendes et rumeurs. Ainsi, selon les sceptiques luttant contre la marée montante des croyances au paranormal, l'étude des rumeurs et des légendes est utile parce qu'elle démontre les processus conduisant à des croyances erronées. Toutefois les liens entre "la réalité» et «les légendes» sont plus complexes et ne peuvent être réduits à des formules simples. Plusieurs ensembles de faits sociaux dérangeants existent et mènent à la création de légendes: les enlèvements d'enfants, les meurtres, l'inceste, etc. Et, inversement, les légendes et les histoires créent des faits et des actes: ce sont les processus d'ostension. 


\section{Bibliographie citée}

Bloch Marc. «Réflexions d'un historien sur les fausses nouvelles de la Grande Guerre", in Mélanges historiques. Paris, SEPVEN, 1963 [première publication 1921] : 41-57.

BONAPARTE Marie. Mythes de guerre. London, Imago, 1946.

BRUNVAND Jan Harold. The Vanishing Hitchbiker 1981, The Choking Doberman 1984, The Mexican Pet 1986, Curses! Broiled Again 1989, The Baby Train 1993. New York: Norton.

CAmPion-Vincent Véronique. "Bébés en pièces détachées: une nouvelle "légende" latino-américaine", Cahiers internationaux de sociologie 93. 1992a: 299-319.

CAmpion-Vincent, Véronique (dir.). Des fauves dans nos campagnes. Légendes, rumeurs et apparitions. Paris: Imago, 1992b.

CAMPION-Vincent Véronique. "Bébés en pièces détachées. Une nouvelle légende latino-américaine, in Le réenchantement $d u$ monde, Paris, L'Harmattan, 1994 : 192-214.

CAmpion-Vincent Véronique et Renard Jean-Bruno (dir.). Rumeurs et légendes contemporaines. Communications 52, 1990.

Campion-Vincent Véronique et Renard Jean-Bruno. Légendes urbaines. Rumeurs d'aujourd'bui. Paris: Payot, 1992.

DAUZAT Albert. "Les faux-bruits et légendes de la guerre", Mercure de France 128, 1918 : 241-262.

DAUZAT Albert. Légendes, prophéties et superstitions de la guerre. Paris, La Renaissance du livre, [1919].

DEGH Linda. "What Is the Legend After All?», Contemporary Legend 1, 1991 : 11-38.

Graux $D^{r}$ Lucien. Les fausses nouvelles de la Grande Guerre. Paris, 1918-1920, 7 vols.

Horne John \& Kramer Alain. German Atrocities, 1914. A History of Denial. New Haven, Yale University Press.
KAPFerer Jean-Noël. Rumeurs. Le plus vieux média du monde. Paris, Seuil, 1987.

JOISTEN Alice \& ChANAUd Robert. «Le loupgarou dans les Alpes françaises ou les degrés du fantastique", in V. CAMPION-VINCENT (dir.), Des fauves dans nos campagnes. Légendes, rumeurs et apparitions. Paris, Imago, 1992 : 115-130.

†Jorsten Charles, Chanaud Robert \& JoIsTEN Alice. "Les loups-garous en Savoie et Dauphiné", Le Monde alpin et rhodanien, 1-4, 1992, Etres fantastiques dans les Alpes. Recueil d'études et de documents en mémoire de Charles Joisten (1936-1981): 19-182.

LE QUeLLEC Jean-Loïc, Alcool de singe et liqueur de vipère... plus quelques autres recettes. Vouillé, Geste Editions, 1991.

MORIN Edgar. La rumeur d'Orléans. Paris, Seuil, 1969.

Ponsonby Arthur. Falsehood in War-Time: Containing an assortment of lies circulated throughout the nations during the Great War. London, G. Allen \& Unwin, 1928.

Ponsonby Arthur. Mensonges et rumeurs en temps de guerre, [Jean PLANTIN, préface, traduction, index et notes]. Saint Genis Laval, Éditions du dragon vert [Cahiers d'bistoire révisionniste 1], 1996.

Reumaux Françoise. Toute la ville en parle: esquisse d'une théorie des rumeurs. Paris, L'Harmattan, 1994.

REUMAUX Françoise. La veuve noire: message et transmission de la rumeur. Paris, Klincksieck, 1996.

VAN LANGENHOVE Fernand. Comment naît un cycle de légendes. Francs-Tireurs et atrocités en Belgique. Paris, Payot, 1917.

VICTOR Jeffrey. "The Sociology of Contemporary Legend. A review of the use of the concept by sociologists", Contemporary Legend 3, 1993: 63-83. 